\title{
Heterostructured silicon-germanium-silicon p-i-n avalanche photodetectors for chip-integrated optoelectronics -INVITED
}

\author{
Daniel Benedikovic ${ }^{1,2, *}$, Leopold Virot ${ }^{3}$, Guy Aubin ${ }^{2}$, Jean-Michel Hartmann ${ }^{3}$, Farah Amar ${ }^{2}$, Xavier Le Roux ${ }^{2}$, Carlos \\ Alonso-Ramos ${ }^{2}$, Milan Dado ${ }^{1}$, Eric Cassan ${ }^{2}$, Delphine Marris-Morini ${ }^{2}$, Jean-Marc Fedeli ${ }^{3}$, Frederic Boeuf ${ }^{4}$, Bertrand \\ Szelag $^{3}$ and Laurent Vivien ${ }^{2}$ \\ ${ }^{1}$ Dept. Multimedia and Information-Communication Technologies, University of Žilina, ,01026 Žilina, Slovakia \\ ${ }^{2}$ UniversitéParis-Saclay, CNRS, Centre de Nanosciences et de Nanotechnologies, 91120 Palaiseau, France \\ ${ }^{3}$ University Grenoble Alpes and CEA, LETI, 38054 Grenoble, France \\ ${ }^{4}$ STMicroelectronics, Silicon Technology Development, 38923 Crolles, France
}

\begin{abstract}
Optical photodetectors are at the forefront of photonic research since the rise of integrated optics. Photodetectors are fundamental building blocks for chip-scale optoelectronics, enabling conversion of light into an electrical signal. Such devices play a key role in many surging applications from communication and computation to sensing, biomedicine and health monitoring, to name a few. However, chip integration of optical photodetectors with improved performances is an on-going challenge for mainstream optical communications at near-infrared wavelengths. Here, we present recent advances in heterostructured silicon-germanium-silicon p-i-n photodetectors, enabling high-speed detection on a foundry-compatible monolithic platform.
\end{abstract}

\section{INTRODUCTION}

Germanium photodetectors integrated with sub-micron silicon waveguides are essential building elements for many applications. This includes sensing, health monitoring, biomedicine or optical interconnects and communications. Silicon-germanium photodetectors are now mature components and their opto-electrical performances are rather compelling. A wide variety of chip-integrated photodetectors have been proposed and demonstrated in recent years [1]. Heterostructured silicon-germanium-silicon photodiodes with a buttwaveguide-coupling scheme and a lateral $p$-i-n electrical junction are promising to detect light on a silicon chip [2-5]. Cross-sectional schematics and an optical microscopy image of such heterostructured p-i-n photodetectors are shown in Fig. 1a and 1b, respectively. This unique integration strategy facilitates the development of compact devices with properly engineered waveguide features. Such photodiodes benefit from improved modal confinement and overall control over the intrinsic region. They are also easier to fabricate [2,3]. Such a photodiode configuration also yields enhanced performances (lower dark-current levels, better responsivities and faster operations) compared to full-germanium architectures [6] or metalsemiconductor-metal [7] alternatives. Moreover, heterostructured p-i-n photodiodes with silicongermanium-silicon heterojunction leverage the maturity of foundry-based silicon processes and germanium epitaxial growth. A robust monolithic integration platform is then obtained, with complex passive and active chip functionalities simultaneously achieved.

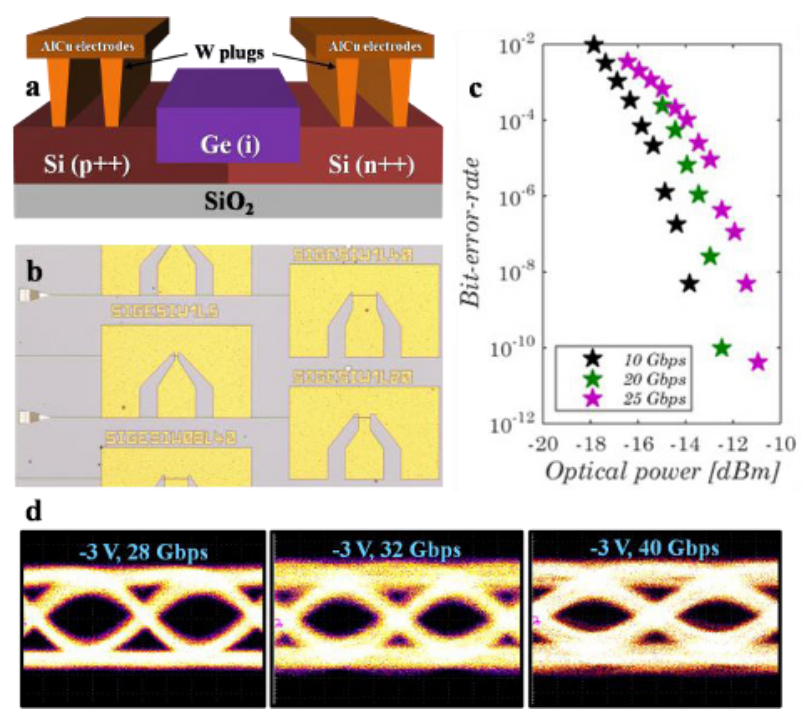

Fig. 1. (a) Schematic view of a p-i-n waveguide photodetector with a lateral silicon-germanium-silicon heterojunction. (b) Optical microscopy image of fabricated p-i-n photodetectors. (c) Bit-error-rate measurements as a function of optical input power for silicon-germanium-silicon photodetector at conventional $10 \mathrm{Gbps}, 20 \mathrm{Gbps}$ and $25 \mathrm{Gbps}$ transmission bit rates and a $3 \mathrm{~V}$ reverse bias. (d) Eye diagram apertures at 28 Gbps, 32 Gbps and 40 Gbps, under the same low-voltage operation (with respective scale adapted). 
Herein, we present recent progress in waveguideintegrated $\mathrm{p}-\mathrm{i}-\mathrm{n}$ photodetectors with lateral silicongermanium-silicon hetero-junctions. Fabricated p-i-n photodiodes were operated under low-voltage (" $p-i-n$ regime") and high-voltage ("avalanche regime") conditions, respectively, delivering high-speed and lownoise detection at a mainstream telecommunication waveband centered at $1.55 \mu \mathrm{m}$.

\section{Opto-electrical performance heterostructured photodetectors}

Heterostructured silicon-germanium-silicon photodetectors of different widths and different lengths were fabricated on 200-mm silicon-on-insulator substrates using industrial-scale silicon processes, aiming at both low-voltage and avalanche operations.

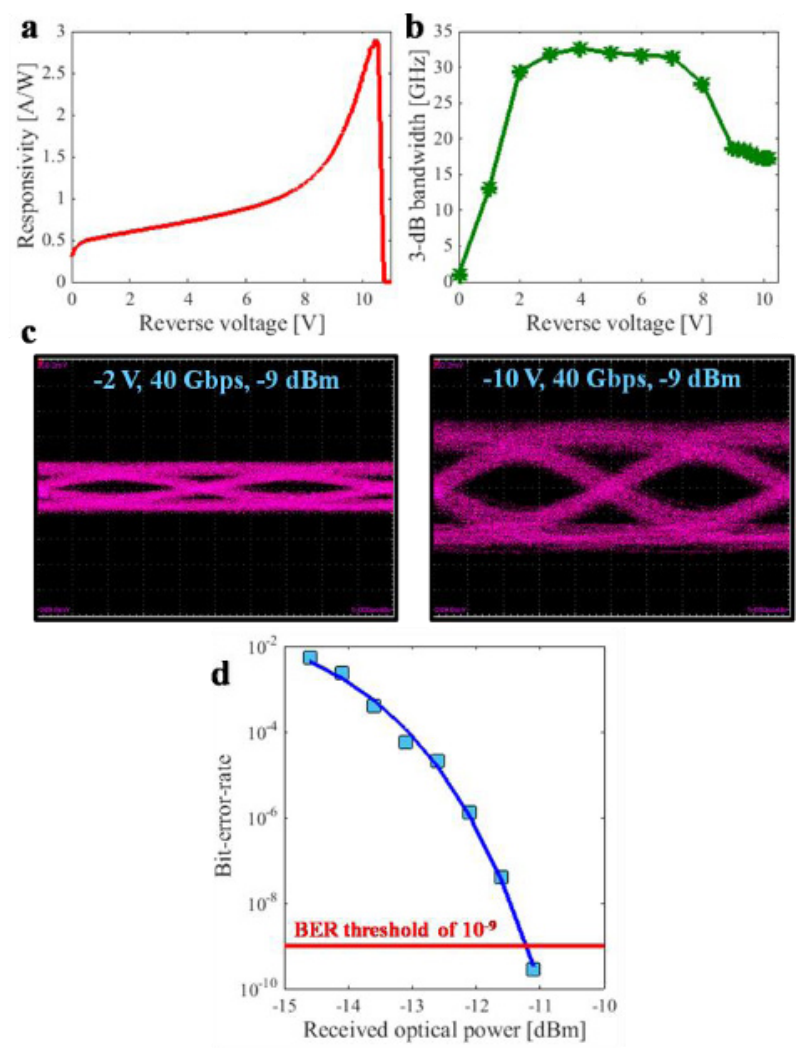

Fig.2. (a) Responsivity-voltage and (b) bandwidth-voltage characteristics of heterostructured silicon-germanium-silicon photodetector operated in an avalanche regime. (c) Output eye diagrams (with same vertical scale) for a $40 \mathrm{Gbps}$ bit rate input signal at $1.55 \mu \mathrm{m}$ carrier wavelength and different reverse bias conditions of $2 \mathrm{~V}$ and $10 \mathrm{~V}$, with static avalanche multiplication gains of 1.4 and 5.5, respectively. (d) Bit-errorrate as a function of a received optical power for a silicongermanium-silicon photodetector operated under an avalanche regime measured with a $40 \mathrm{Gbps}$ signal, a reverse operating voltage of $10 \mathrm{~V}$ and an avalanche net-light gain of 7.5.

Under low-voltage operation, silicon-germanium-silicon photodetectors exhibited dark currents in the $5 \mathrm{nA}$ to 150 $\mathrm{nA}$ range for the smallest up to the largest device geometries [5]. The corresponding dark-current densities were thus in the $0.404 \mathrm{~A} / \mathrm{cm}^{2}$ to $0.808 \mathrm{~A} / \mathrm{cm}^{2}$ range. Device responsivities of $0.20 \mathrm{~A} / \mathrm{W}$ and $1.2 \mathrm{~A} / \mathrm{W}$ were measured at $0.5 \mathrm{~V}$ reverse bias for the shortest and the longest photodetector designs $[3,4]$. Interestingly, the photodiode responsivities reached their maxima at reverse voltages as low as $0.5 \mathrm{~V}$. These trends were consistently observed on all tested devices. Beyond a 0.5 $\mathrm{V}$ reverse bias, the photo-responsivity remained virtually flat, with small voltage dependence [4]. The strong builtin electrical field at low bias states showed up the great ability of such heterostructured $p$-i-n devices to sweep out the majority of the electron-hole pairs within their lifetime. Moreover, the $-3 \mathrm{~dB}$ bandwidth was inversely proportional to the intrinsic region width, while the bandwidth remained unchanged for devices of different lengths and a fixed width [4,5]. This may indicate that the frequency response of the heterostructured $p-i-n$ photodiode was not limited by the resistancecapacitance-delay. On the other hand, the device speed limit stemmed mainly from the transit time of photocarriers. Measured $-3 \mathrm{~dB}$ bandwidths were in the $7 \mathrm{GHz}$ to $35 \mathrm{GHz}$ range [5].

Figure 1c shows bit-error-rate results obtained for $1 \mu \mathrm{m}$ wide by $40 \mu \mathrm{m}$ long silicon-germanium-silicon photodiode. Measurements were carried out with a pseudo-random bit sequence (PBRS) at $10 \mathrm{Gbps}, 20$ Gbps, and 25 Gbps transmission bit rates, yielding optical power sensitivities of $-14 \mathrm{dBm},-13 \mathrm{dBm}$, and -11 $\mathrm{dBm}$ for a $10^{-9}$ error-rate threshold [4]. In addition, Fig. 1d illustrates low-voltage operations, precisely here at 3 $\mathrm{V}$ reverse bias with electrical eye diagram apertures that remained open at $28 \mathrm{Gbps}, 32 \mathrm{Gbps}$, and $40 \mathrm{Gbps}$. Such results pave a way for an on-chip detection of high-speed optical signals using silicon-germanium-silicon p-i-n photodetectors driven with low operating voltages.

However, the photo-responsivity is limited in conventional $\mathrm{p}-\mathrm{i}-\mathrm{n}$ photodiodes, because of a reduced external quantum efficiency. Optical power sensitivities are then modest for high-bit-rate optical signals and additional amplification circuits are mandatory for use in transmission networks and systems [1].

Avalanche photodetectors are very good alternatives to such device structures, as they can tackle many challenges. Avalanche photodetectors indeed leverage, under generated high electrical fields, an impact ionization effect, resulting in a significant internal multiplication gain. This internal gain is then harnessed to improve the diode performances in terms of device sensitivity, operation speed and generated noise [8-15].

Figures $2 \mathrm{a}$ and $2 \mathrm{~b}$ show the photo-responsivity and the $3 \mathrm{~dB}$ bandwidth as a function of reverse bias for a 0.5 $\mu \mathrm{m}$ wide and $40 \mu \mathrm{m}$ long silicon-germanium-silicon photodetector. Operating the silicon-germanium-silicon $\mathrm{p}-\mathrm{i}-\mathrm{n}$ photodiode in an avalanche regime substantially improves its photo-responsivity, from a nominal value of $0.5 \mathrm{~A} / \mathrm{W}$ at low reverse voltages up to almost $3 \mathrm{~A} / \mathrm{W}$ close to avalanche breakdown. This improvement corresponds to an avalanche multiplication net-light gain 
of about 6. The transition from an absorption regime towards an avalanche regime is otherwise rather gentle. On the other hand, the $-3 \mathrm{~dB}$ bandwidth of the device is reduced from its maximum of about $33 \mathrm{GHz}$ (for $3 \mathrm{~V}$ up to $7 \mathrm{~V}$ reverse bias) down to $16 \mathrm{GHz}$ (for reverse bias of $10 \mathrm{~V})$. This device bandwidth drop is caused by the avalanche built-up time. Operating the diode in an avalanche regime provides the means to improve the signal detection. This is clearly shown in Fig. 2c. Here, electrical eye diagrams for $40 \mathrm{Gbps}$ optical signals and two different bias conditions ( $2 \mathrm{~V}$ with a net-light gain of only 1.4 and $10 \mathrm{~V}$ with a net-light gain of 5.5) are depicted.

Moreover, silicon-germanium-silicon photodetectors are also capable of high-speed and low-noise operation, with a gain-bandwidth product up to $210 \mathrm{GHz}$ and a low excess noise factor with an effective ionization ratio of about 0.25 [15]. The low noise characteristics of silicongermanium-silicon photodetectors are attributed to the strong impact ionization processes occurring close to the silicon-germanium interfaces. In mainstream fullgermanium $\mathrm{p}$-i-n photodetectors, such processes take place in germanium, hindering a low-noise operation. Indeed, silicon-germanium-silicon photodiodes benefit from the low noise properties of silicon, with an extremely low ionization ratio. The intrinsic avalanche multiplication decreases the avalanche excess noise, and thus reducing the effective ionization coefficient through the dead space effect.

Finally, Fig. 2d shows bit-error-rate measurements versus received optical power for 40 Gbps optical signals. An optical power sensitivity of about $-11 \mathrm{dBm}$ was obtained with such a silicon-germanium-silicon photodetector for an error-rate level of $10^{-9}$. Further device sensitivity improvements were prevented by the large dark current close to the avalanche breakdown. The dark current at the avalanche breakdown of $\sim 11 \mathrm{~V}$ indeed reached $600 \mu \mathrm{A}$.

\section{Conclusions}

In this work, we presented recent achievements on chipintegrated silicon-germanium-silicon lateral p-i-n photodetectors. Devices were capable of $40 \mathrm{Gbps}$ onchip detection in low-voltage ("p-i-n regime") and highvoltage ("avalanche regime") conditions. Devices capitalized on the combination of butt-waveguide coupling with lateral silicon-germanium-silicon p-i-n junctions. Devices thus had a reduced footprint, a definitely reduced fabrication complexity and significantly improved opto-electrical performances. These results open up opportunities to deploy silicongermanium-silicon photodetectors into high-speed and cost-effective on-chip networks, targeting many emerging photonic applications in health monitoring, biomedicine, data centres, or optical interconnects.

\section{Acknowledgement}

This work was supported by the Slovak Research and Development Agency under the project APVV-17-0631 CONSENS and PP-COVID-20-0100: DOLORES.AI: The pandemic guard system.

\section{References}

1. D. Benedikovic, et al., Nanophotonics10, 1059 (2021)

2. H. Chen, et al., Opt. Express 24, 4622 (2016)

3. L. Virot, et al.,Opt. Express 25, 19487 (2017)

4. D. Benedikovic, et al., Photon. Res. 7, 437 (2019)

5. D. Benedikovic, et al., IEEE J. Quantum Electron. 56, 8400409 (2020)

6. J. H. Nam, et al., Opt. Express 23, 15816 (2015)

7. H. Pan, et al., Opt. Express 20, 18145 (2012)

8. S. Assefa, et al., Nature 464, 80 (2010)

9. Y. Kang, et al., Nat. Photonics 3, 59 (2009)

10. L. Virot, et al., Nat Commun. 5, 4657 (2014)

11. H. T. Chen, et al., Opt. Express 23, 815 (2015)

12. Z. Huang, et al., Optica3, 793 (2016)

13. X. Zeng, et al., Optica6, 772 (2019)

14. S. A. Srinivasan, et al., IEEE/OSA J. Light. Technol. 38, 3044 (2020)

15. D. Benedikovic, et al., Optica 7, 774 (2020) 